\title{
Multiple Lacunar Infarcts in Paroxysmal Nocturnal Hemoglobinuria
}

\author{
Alex Tiburtino Meira, MR, ${ }^{*}$ Gabriel Sampaio Froehner, $\mathrm{MR},+$ \\ André Petean Trindade, MD, PhD, $\ddagger$ Silméia Garcia Zanati Bazan, MD, PhD, $§$ \\ Gabriel Pereira Braga, MD, PhD, $\|$ and Rodrigo Bazan, $\mathrm{MD}, \mathrm{PhD} \|$
}

\begin{abstract}
Paroxysmal nocturnal hemoglobinuria $(\mathrm{PNH})$ is a rare acquired disease characterized by clonal hematopoietic stem cell disorder, with increased mortality and morbidity. Venous thrombosis is the most common cause of mortality in PNH. The relationship between $\mathrm{PNH}$ and cerebrovascular disease is unclear; few cases are reported in the literature, most of them related to cerebral venous thrombosis; In $\mathrm{PNH}$ the involvement of intracranial and extracranial arterial sites is very rare. We report a case of a 49-year-old woman who has a medical history of diabetes mellitus, hypertension, and $\mathrm{PNH}$ and presented multiple lacunar strokes in a routine consultation with a hematologist. A brain computed tomography (CT) scan showed lacunar infarcts, and magnetic resonance image showed acute ischemic stroke, multiple territory lacunar infarctions, and focal area of microbleeds in gradient echo sequence. A CT angiography showed V3 and V4 branches of the left vertebral artery occluded by a thrombus, and the posterior inferior cerebellar artery occluded, whereas the carotid system was normal. We discuss the presentation and physiopathology of stroke in $\mathrm{PNH}$ and other cases reported in the literature review. Key Words: Stroke-paroxysmal nocturnal hemoglobinuria-magnetic resonance-cerebrovascular disease-hematopoietic disorder.

(C) 2017 National Stroke Association. Published by Elsevier Inc. All rights reserved.
\end{abstract}

\footnotetext{
From the *Medical Resident at Clinics Hospital, Botucatu Medical School-UNESP, São Paulo State University, São Paulo, Brazil; +Medical Resident at Clinics Hospital, São Paulo University, Ribeirão Preto, São Paulo, Brazil; łDepartment of Tropical Diseases and Diagnostic Imaging; §Department of Internal Medicine; and \|Department of Neurology, Botucatu Medical School-UNESP, São Paulo State University, São Paulo, Brazil.

Received October 23, 2016; revision received March 21, 2017; accepted July 5, 2017.

Address correspondence to Rodrigo Bazan, MD, PhD, Universidade Estadual Paulista Júlio de Mesquita Filho, Faculdade de Medicina de Botucatu, Departamento de Neurologia e Psiquiatria, R. Professor Montenegro, Distrito de Rubião Júnior, s/nº 18618-970, Botucatu, SP, Brazil. E-mail: bazan.r@terra.com.br.

1052-3057/\$ - see front matter

(C) 2017 National Stroke Association. Published by Elsevier Inc. All rights reserved.

http:/ /dx.doi.org/10.1016/j.jstrokecerebrovasdis.2017.07.001
}

\section{Introduction}

Stroke is one of the leading causes of death worldwide and is a major cause of adult disability. Paroxysmal nocturnal hemoglobinuria (PNH), first described in 1882 by Dr. Paul Strübing, ${ }^{1}$ is a rare acquired disorder in which a clonal hematopoietic stem cell disorder occurs: mutations in an X-linked gene-phosphatidylinositol glycan complementation class A gene. ${ }^{2}$ It leads to intravascular hemolysis, which is responsible for anemia, hemoglobinuria, thrombosis, pulmonary hypertension, and renal impairment. Common presentation is hemolytic anemia, bone narrow failure, and thrombosis (the most severe complication and main cause of mortality). ${ }^{3}$ Venous thrombosis is more common than arterial. ${ }^{4}$ The most common sites of thrombosis are intra-abdominal as hepatic, portal, mesenteric, splenic veins and cerebral veins such as sagittal and cavernous sinus. ${ }^{5}$ The relationship between $\mathrm{PNH}$ and cerebrovascular 
disease is unclear; few cases are reported in the literature, most of them related to cerebral venous thrombosis, but it is responsible for $25 \%$ of deaths in this disease. ${ }^{6}$ The involvement of intracranial and extracranial arterial sites is very rare. ${ }^{4}$ We report a case of a young woman with a medical history of PNH who presented stroke sequelae in a routine consultation with a hematologist.

\section{Case Report}

A 49-year-old woman presented hemifacial paresis of sudden onset days before in a routine consultation with a hematologist. She had a previous medical history of diabetes mellitus and well-controlled hypertension, and a 30-year history of PNH. She had Budd-Chiari syndrome (BCS) 30 years ago, which was found, upon investigation, to be hemolytic anemia and thrombocytopenia, which drew attention to PNH. Flow cytometry was performed and showed $42 \%$ neutrophils, $8 \%$ monocytes, and $24 \%$ erythrocytes with CD59 deficiency. She presented chronic liver disease as a sequel to this episode. In the subsequent years, she had multiple deep venous thrombosis, one in each leg and one in the right arm. Fifteen years ago, she used an anticoagulant for 2 years.

On admission to the neurology ward, she has mild left hemiparesis, which was noticed by the patient 9 months ago, hypoesthesia in the left ophthalmic branch of the trigeminal nerve, and mild lateropulsion to the left. The previous modified Rankin Scale score was 2. The National Institutes of Health Stroke Scale score was 3. Laboratorial exams showed hemoglobin, $9.1 \mathrm{~g} / \mathrm{dL}$; hematocrit, $28.1 \%$, with anisocitosis; platelets, $14.000 / \mathrm{mm}^{3}$; leukocytes, $3.800 / \mathrm{mm}^{3}$; reticulocytes, $2.72 \%$; lactate dehydrogenase, $1185 \mathrm{U} / \mathrm{L}$; glycated hemoglobin, 7.5\%; and international normalized ratio, 1.21. Others were normal (blood urea, creatinine, sodium, potassium, aspartate aminotransferase, alanine aminotransferase, bilirubin, serologies [syphilis, Chagas disease, HIV], thyroid function, total cholesterol, tryglicerides and fractions, screening for stroke in a young patient). A brain computed tomography (CT) scan showed 2 new areas of hipodensity compared with previous CT of 3 months prior, and a brain magnetic resonance imaging (MRI) demonstrated acute and chronic ischemic stroke areas, and the focal area of microhemorrhage (Fig 1). On Transcranial Doppler, the left vertebral artery seemed to be hypoplastic, or resistance increased downstream. A CT angiography showed the V3 and V4 branches of the left vertebral artery occluded by a thrombus, and the posterior inferior cerebellar artery occluded, whereas the carotid system was normal. A transthoracic echocardiography revealed concentric left ventricular hypertrophy and tricuspid regurgitation; then, a transesophageal echocardiography

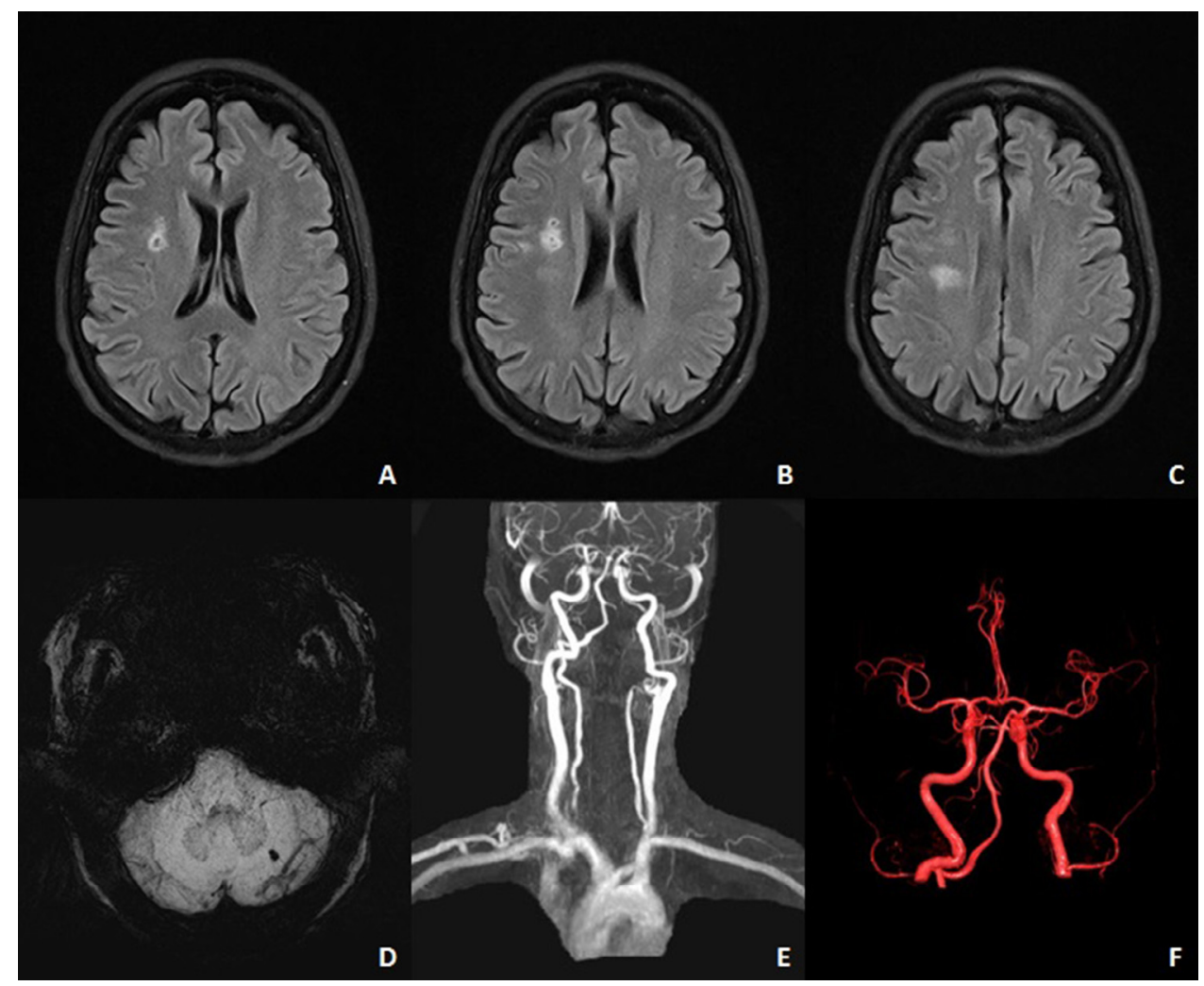

Figure 1. Patient's MRI and magnetic resonance angiography. (A-C) MRI. Fluid-attenuated inversion recovery demonstrating hyperintensity areas in the region of right parietal and frontal lobes, lesions compatible with acute ischemic lacunar infarction. (D) Gradient ECHO showing focal area of microbleeding in the left cerebellar hemisphere. (E-F) Three-dimensional time-of-flight showed left vertebral occlusion in the V3 and V4 segments. Abbreviation: MRI, magnetic resonance imaging. 
was negative for the passage of microbubbles (right-toleft shunt) or intracavitary thrombi. There were no apparent signs of vasculitis or arterial dissection on the magnetic resonance angiography.

During hospitalization, there was no recurrence of stroke, and the patient had pneumonia, which was properly treated with antibiotics. She was discharged with acetylsalicylic acid $200 \mathrm{mg}$ daily despite thrombocytopenia. No anticoagulant was prescribed due to her altered hepatic function. The modified Rankin Scale score was 2. The National Institutes of Health Stroke Scale score was 3.

Nowadays, the patient is under hematologist, hepatologist, and neurologist supervision, and the deficits are the same on the discharge. She is under the juridical process for receiving eculizumab treatment.

\section{Discussion}

Although this young patient was diabetic and hypertensive, the comorbidities were well controlled. Her social history is remarkable. She has never smoked or drunk alcoholic drinks.

This case shows us the importance of medical history when accompanying a stroke patient. The past history of $\mathrm{PNH}$ in this case was remarkable: she already had 2 kinds of complications of the disease- both in the venous system (Budd-Chiari syndrome and three deep venous thrombosis). This time, the arterial system was affected: multiple lacunar strokes, arterial thrombus, and microhemorrhage. This has a major importance because intracerebral veins are more commonly affected than arteries, which can be noticed in the literature: only a few cases of cerebrovascular involvement are in the arterial circulation., ${ }^{4,7}$

The most recent study regarding PNH-related cerebral venous or arterial thrombosis showed that, from 35 cases, the commonest finding is cerebral venous thrombosis $(66 \%)$, with superior sagittal sinus being the most frequently involved site. The arterial site is less common $(42.9 \%$, including cases involving both arterial and venous sites), with the middle cerebral artery the most common site of arterial involvement. ${ }^{4}$

CT and MRI showed involvement of small and large vessels. Lacunar infarcts and gliosis areas in the frontal lobe, thrombosis of the left vertebral artery, and microbleeds in the cerebellum were found in this case. This presentation on $\mathrm{PNH}$ is rare.

Thrombosis in $\mathrm{PNH}$ is multifactorial. It involves the absence of complement regulatory proteins in the surface of platelets, low levels of nitric oxide, the generation of inflammatory cytokines, and defective fibrinolysis. All contribute to a prothrombotic state. ${ }^{2}$ Peripheral blood flow cytometry detecting the absence or severe deficiency of the phosphatidylinositol glycan complementation class A gene on more than one lineage is diagnostic of PNH. The larger the percentage of PNH cells, the more probable is the risk of thrombosis in the patient, especially with granulocyte clone sizes larger than $50 \% .^{5}$ Beyond this rate, the cumulative incidence rate of thrombosis is $34.5 \%$, and below this rate, it is $5.3 \%){ }^{9}$

Thrombosis is the leading cause of death from $\mathrm{PNH}$ and should be treated promptly with anticoagulation and/or thrombolytic therapy, which depends on the site of the thrombus. ${ }^{7}$ Complement inhibition with eculizumab is cornerstone of the treatment: major impact in the prevention of thrombosis as well as its management in this disease. ${ }^{10}$ This medication is the only U.S. Food and Drug Administration-approved therapy for the disease; it's a monoclonal antibody that binds to $\mathrm{C} 5$ and blocks terminal complement. In this way, the monoclonal antibody formation is inhibited, which compensates CD59 deficiency (9 but not CD55 deficiency); it explains how the medication reduces intravascular hemolysis. Headache is the most common side effect, and Neisseria life-threatening infections are the most dangerous ones. ${ }^{1}$ For patients with therapeutic failure, despite eculizumab, bone marrow transplantation is the only cure for $\mathrm{PNH} .^{10}$

This patient had not used such medication before the stroke, but today she is under the juridical process in order to receive this medication. It's possible yet, the curative choice.

\section{Conclusion}

In stroke in young patients, $\mathrm{PNH}$ is a rare but feasible cause. It commonly presents as venous thrombosis; however, in some cases it can present as arterial involvement, and this should be remembered. Suggesting periodic screening in patients diagnosed with $\mathrm{PNH}$ is plausible. Studies are needed to demonstrate whether there is benefit in performing routine CT or MRI in these patients. But we recommend routine consultation with a neurologist for all $\mathrm{PNH}$ patients, especially for those with $\mathrm{PNH}$ granulocyte clone sizes larger than $50 \%$.

\section{References}

1. Brodsky RA. Paroxysmal nocturnal hemoglobinuria. Blood 2014;124:2804-2811.

2. Hill A, Kelly RJ, Hillmen P. Thrombosis in paroxysmal nocturnal hemoglobinuria. Blood 2013;121:4985-4996.

3. Grotta JC, Manner C, Pettigrew LC, et al. Red blood cell disorders and stroke. Stroke 1986;17:811-817.

4. Poulou LS, Vakrinos G, Pomoni A, et al. Stroke in paroxysmal nocturnal haemoglobinuria: patterns of disease and outcome. Thromb Haemost 2007;98:699701.

5. Parker C, Omine M, Richards S, et al. Diagnosis and management of paroxysmal nocturnal hemoglobinuria. Blood 2005;106:3699-3709.

6. Tiu R, Yacoub H, Maciejewski J, et al. Recurrent ischemic stroke in paroxysmal nocturnal hemoglobinuria: paroxysmal nocturnal hemoglobinuria or missed patent foramen ovale? J Stroke Cerebrovasc Dis 2009;18:409410. 
7. Samadder NJ, Casaubon L, Silver F, et al. Neurological complications of paroxysmal nocturnal hemoglobinuria. Can J Neurol Sci 2007;34:368-371.

8. Meppiel E, Crassard I, Peffault de Latour R, et al. Cerebral venous thrombosis in paroxysmal nocturnal hemoglobinuria: a series of 15 cases and review of the literature. Medicine (Baltimore) 2015;94:362.
9. Malato A, Saccullo G, Lo Coco L, et al. Thrombotic complications in paroxysmal nocturnal haemoglobinuria: a literature review. Blood Transfus 2012;10:428-435.

10. Hill A, Kelly RJ, Kulasekararaj AG, et al. Eculizumab in paroxysmal nocturnal hemoglobinuria $(\mathrm{PNH})$ : a report of all 153 patients treated in the UK. Blood 2012;120: 3472 . 